BIO Web of Conferences 1, 00022 (2011)

DOI: $10.1051 /$ bioconf $/ 20110100022$

(C) Owned by the authors, published by EDP Sciences, 2011

\title{
Movement Sonification: Audiovisual benefits on motor learning
}

\author{
Alfred Effenberg ${ }^{*}$ Ursula Fehse $^{*}$ Andreas Weber ${ }^{\ddagger}$ \\ (*)Institute of Sport Science, University of Hanover, Germany \\ $\left({ }^{*}\right)$ Institute of Informatics II, University of Bonn, Germany \\ E-mail:alfred.effenberg@sportwiss.uni-hannover.de,ursula.fehse@sportwiss.uni-hannover.de.
}

\begin{abstract}
Processes of motor control and learning in sports as well as in motor rehabilitation are based on perceptual functions and emergent motor representations. Here a new method of movement sonification is described which is designed to tune in more comprehensively the auditory system into motor perception to enhance motor learning. Usually silent features of the cyclic movement pattern "indoor rowing" are sonified in real time to make them additionally available to the auditory system when executing the movement. Via real time sonification movement perception can be enhanced in terms of temporal precision and multi-channel integration. But beside the contribution of a single perceptual channel to motor perception and motor representation also mechanisms of multisensory integration can be addressed, if movement sonification is configured adequately: Multimodal motor representations consisting of at least visual, auditory and proprioceptive components - can be shaped subtly resulting in more precise motor control and enhanced motor learning.
\end{abstract}

\section{Introduction}

Visual feedback as well as visual instruction both have a long tradition in the field of motor learning in sports. After using film for many decades video has become very popular for supporting motor learning in sports starting broadly in the seventies of the last century. Video technology has been used on many different kinds of sports like figure skating, apparatus gymnastics or high diving. And indeed it is helpful for a novice observing a video of a "model athlete" performing the movement pattern again and again to support motor learning: With the mirror neuron system neuroscientific research has revealed a visual key mechanism participating in motor learning [1]. But mirror neurons are not only working within the visual domain: There is additional evidence that mirror neurons also respond to auditory input as well as to audiovisual input [2,3]. And even actual work on motor learning describes motor representations as multimodal integrative representations [4].

Consequently the last years a growing number of research has been realized on enhancing motor control - and first studies also on motor learning - by means of audiovisual information $[5,6]$. Here a method of movement sonification is described generating continuous real time sonification of kinematic and dynamic movement features of indoor rowing. Resulting movement sounds contain structural analogies to visual and proprioceptive percepts enhancing audiovisual and audio-proprioceptive integration [7] and enabling the tuning of the multimodal percept [8]. This kind of movement sonification has been used here in a well controlled experiment on motor learning with novices demonstrating that motor learning is indeed enhanced significantly by means of real time movement sonification compared to a visual learning group and also to an audiovisual group with natural movement attended sounds.

\section{Method: 4-channel real time sonification of indoor rowing}

To achieve structural analogy to visual and proprioceptive percepts two kinematic (visual analogy) and two dynamic (proprioceptive analogy) movement features of the indoor rowing motion are sonified. Four sensor systems were applied to the rowing ergometer type "Concept2": A resistance strain gauge for grip force (GF), two sensors for footrest force (FF) and two incremental encoders each for grip pull out length (GP) and sliding seat position (SP). All four parameters were recorded with $100 \mathrm{~Hz}$, for footrest force the sum of the two sensor streams was computed.

Kinematic and dynamic data were recorded by FES-Software $^{1}$ and transmitted via LabVIEWSoftware $^{2}$ to sonification-software MLmini ${ }^{3}$. All parameters are shown in Fig. 1.

\footnotetext{
1 FES: Institute for Research and Development of Sports Equipment, Berlin.

${ }^{2}$ LabVIEW software, National Instruments.
}

This is an Open Access article distributed under the terms of the Creative Commons Attribution-Noncommercial License 3.0, which permits unrestricted use, distribution, and reproduction in any noncommercial medium, provided the original work is properly cited. 


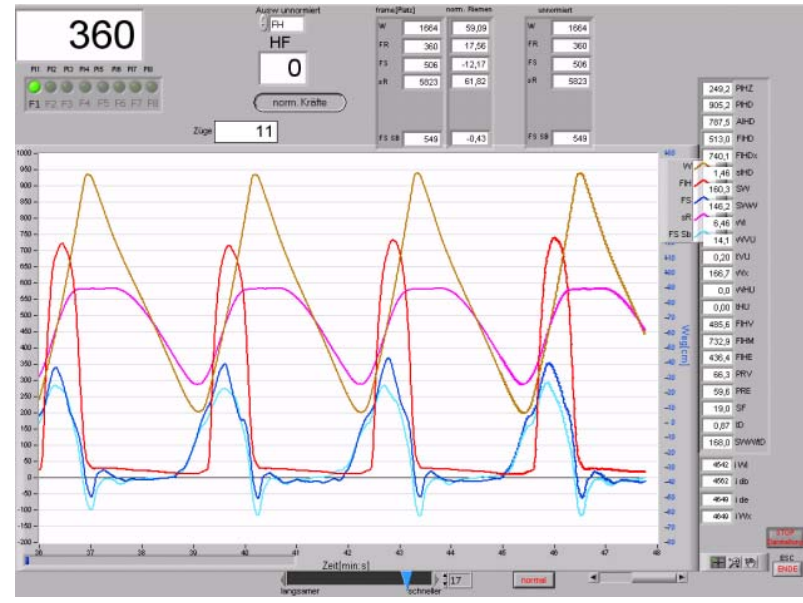

Fig. 1: Four cycles of rowing; grip force (red), bilateral footrest forces (blue), grip pull-out length (yellow) and sliding seat position (magenta).

Movement data were systematically mapped on sound features: Each data stream was used to modulate frequency and partially also amplitude of a certain midi sound.

- Channel 1: Grip pull out length: Accordion, double octave C4-C6, amplitude modulation.

- Channel 2: Grip force: Violin, C2-E5, fixed amplitude.

- Channel 3: Footrest force: French Horn, C3G4, amplitude modulation.

- Channel 4: Sliding seat position: Bag Pipe, E1-C3, fixed amplitude.

Grip pull out, grip force and footrest force were represented continuously. For both force parameters a muting level was defined for values near around zero as well as for negative values to avoid oscillating sound for fast changing forces near around zero: So forces could only be acoustically perceived when they were also clearly perceivable on proprioception. In contrast to the three continuously transformed parameters the sliding seat position was sonified eventrelated: Only at maximum and minimum position on the sliding bar a sound was turned on generating a basic rhythm of the motion. Independently of realized absolute forces, absolute grip pull out length and absolute sliding seat position of a single subject the frequency interval was chosen in a manner that maximum and minimum of individual data streams were related to an identical frequency for each participant in each training session. Though

3 Software MLmini, University of Bonn, Institut of Computer Graphics, Prof. Andreas Weber. sonification of the rowing model was produced the same way this kind of normalization enables participants to generate the same sound pattern (frequencies, amplitudes, timbres) as the model, independently of individual physical strength abilities and body anthropometry.

\subsection{Participants}

The study on motor learning of the rowing technique was conducted with male novices without any rowing experience. 48 volunteers participated in the experiment (mean age $=22,8 \pm 5,0$ ). All participants showed normal or corrected-to-normal vision and normal hearing ${ }^{4}$. They all gave their written consent (their parents resp.) to participate in the study, which was performed in accordance to the Helsinki declaration. Total sample was divided into three samples, carefully parallelized by the initial technical skill level and age. The three samples ran the same training procedure, each with a different kind of information in terms of instruction and real-time feedback.

- Visual condition (V): Sample V was only treated with video information.

- Natural audiovisual condition (AVnat): Sample AVnat was treated with video and natural motion attended sounds of the ergometer windwheel, sliding seat and grip chain pull out.

- Sonified audiovisual condition (AVsoni): Sample AVsoni was treated with video and 4channel movement sonification.

\subsection{Procedure}

Participants were instructed to observe the rowing technique of the model carefully and to approach their own technique as close as possible to the model's. Participants trained for three weeks, two times each week. A training session contained of five blocks, 50 cycles each. Before and after each block the instruction video was presented, feedback was given for 10 cycles in the middle of each block. After the training phase of three weeks there was a break of three weeks with no rowing at all followed by a retention test without and with instruction via the rowing model.

\footnotetext{
4 Standard vision and hearing test: HTTS, Version $2.10,00115.04711$.
} 
The instruction video showed the motion of a junior world champion ${ }^{5}$ performing on the same rowing ergometer "Concept2", Fig. 2 shows all stimuli. movement sonification based on kinematic and dynamic data resulted in a multichannel continuous motion sound which was presented via headphones.

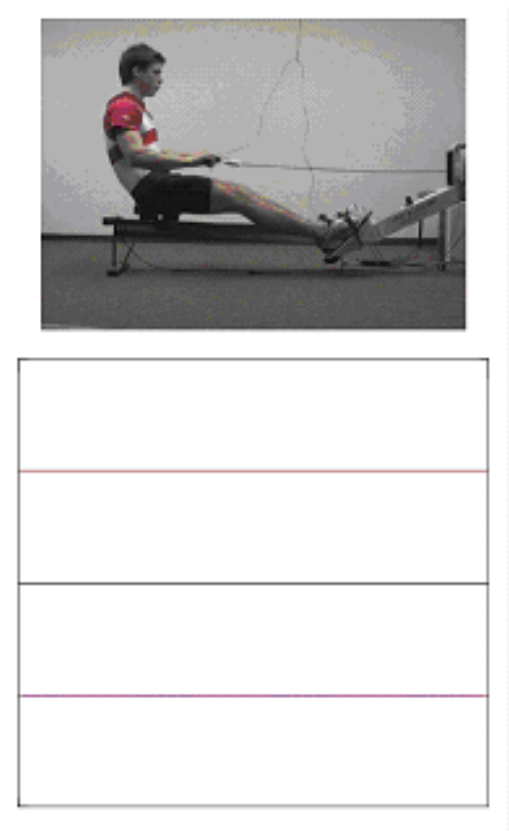

a)

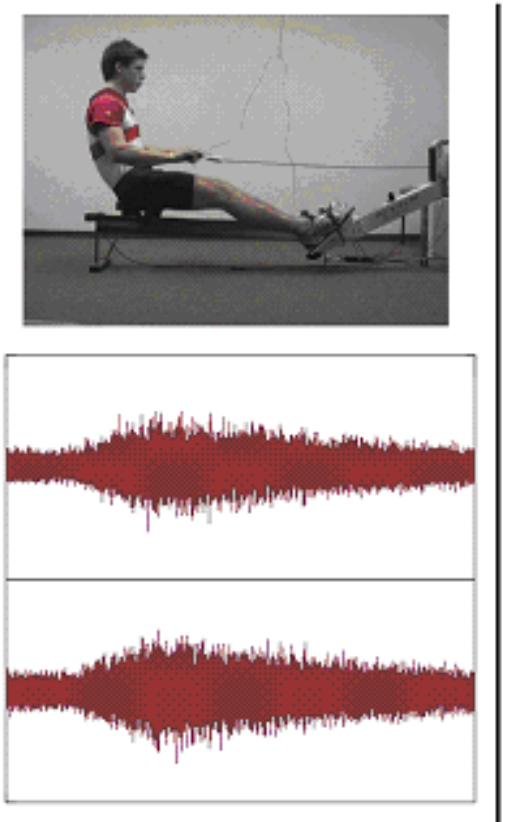

b)
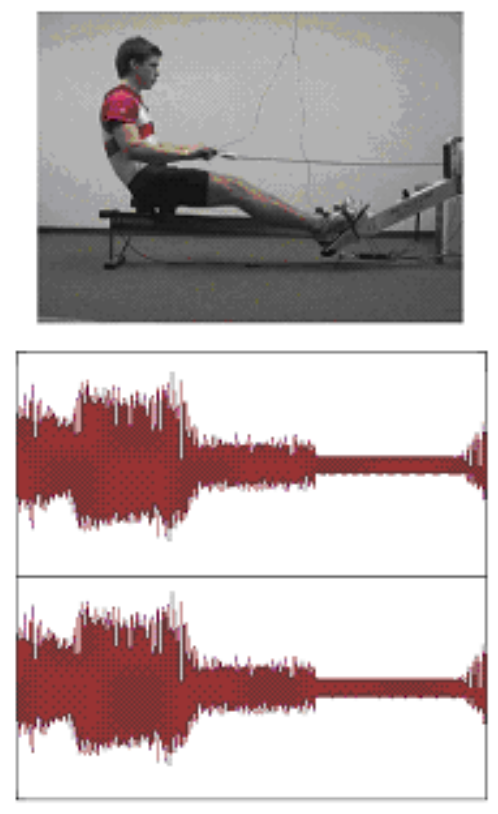

c)

Fig. 2: Visual and auditory stimuli under the three experimental conditions: Frame of instruction video + sound pressure level of soundtrack; a) sample V, b) sample AVnat and c) sample AVsoni.

The videosequence contained 10 cycles of rowing. As feedback, participants observed their own rowing for ten cycles. Depending on the treatment they heard no motion attended sounds, their natural motion attended sounds or their own movement sonification in real time additionally to the video projection. Videos were projected on a big screen in front of the rowing ergometer. For instruction and feedback videos were animated with the Sony Video Capture 6.0b Software.

To mask natural motion attended sounds all participants heard noise (sea rushing) via headphones while there was no auditory instruction or feedback.

Auditory stimuli were presented via headphones. For sample AVnat the sound of the ergometer windwheel, sliding seat and grip chain pull out were taped with a directional microphone ${ }^{6}$ and transmitted via headphones ${ }^{7}$. For AVsoni sample real time

\footnotetext{
${ }^{5}$ Eric Johannesen, junior world champion 2005, four with coxswain $(4+)$.

${ }^{6}$ Type "Behringer ECM 8000".

${ }^{7}$ Type "beyer dynamic DT 100".
}

For data analysis cycles $31-40$ of each 50 cycles training block were selected. An average curve was computed for each of the four raw data streams. In a second step data were normalized to eliminate differences in body size and individual strength. Grip pull out and sliding seat position data were normalized on values between 0 and 1 , grip force and footrest force data were only divided by the particular maximal value to maintain algebraic sign of measured values.

Using the dynamic-time-warping (DTW) algorithm [9] we calculated the distance values between the model's technique and participants' individual technique for each of the four parameters. To consider the rate of force additionally a force index (FI) was built in terms of the average grip force during drive phase divided by the maximum force value (MFV). Maximum force value consisted of the mean of maximum forces (sum of legpress \& row machine) collected in force pre- and posttest: 
$\mathrm{MFV}=(($ maximum force from legpress pretest + row machine pretest $)+$ (maximum force from legpress posttest + row machine posttest)) / 2

A combination of the four distance values with the force index allows to build a general distance value (GDV, see Fig. 3) for each block (five blocks a training session multiplied by six training sessions + pretest, retention test):

$\mathrm{GDV}=5 \mathrm{x}$ distance value $\mathrm{GF}+$ distance value $\mathrm{FF}$ + distance value GP + distance value $\mathrm{SP}-0.5 \times$ FI

Fig. 3: Formula to compute the general distance value (GDV). GF: Grip force, FF: Footrest force, GP: Grip pull out length, SP: Sliding seat position, FI: Force index. Distance value of the grip force is weighted fivefold, other distance values one-way, half of force index is subtracted.

Additionally, a variability coefficient was computed by dividing the standard deviation of mean energy expended during grip pull out by its mean, to quantify the stability of motion. Also duration of drive phase was computed to check the ratio between drive and recovery phase.

\section{Results and discussion}

A first preliminary data analysis revealed differences for the AVsoni sample in terms of faster and more precise learning compared to both other samples. And also for the retention test this effect remained stable, indicating that indeed learning has been realized. In the study it was somehow surprising, that also a significant difference between AV soni and the second sample AVnat (hearing motion sounds in terms of natural attended sounds of the rowing ergometer) was found. It might have been because the movement sonification was structured more concisely than the natural sounds - as could be seen in Fig. 2. But this has to be demonstrated once more.

These findings would be in line with already published findings related to motor perception, motion assessment and motor reproduction as shown for the counter movement jump [10]. There is growing evidence that movement sonification is efficient on motor control and there is first evidence that it is also efficient on motor learning. Motor perception can be enhanced by concordant multimodal information. Indeed there is some subsidiary evidence from a fMRI study that multimodal information (movement video and movement sonification based on a dynamic movement parameter) is addressing multisensory integration sites of the central nervous system additionally [11] and multisensory percepts (movement visualization and movement sonification based on kinematic movement parameters) are processed within different circuits [12, in review]. But currently neurophysiological evidence from our own workgroup is restricted to motor perception. Now it has to be shown, that also emergent motor representations are enhanced by the means of additional movement sonification.

\section{Acknowledgements}

Special thanks to Heinz Mechling (Cologne) for helping initiating this work. This work was financially supported by the Federal Institute of Sport Science, Bonn, Germany, No. 39-WOB.

\section{References}

[14] G. Rizzolatti, L. Fogassi, and V. Gallese. Neurophysiological mechanisms underlying the understanding and imitation of action. Nature Reviews Neuroscience, 2(9), 661-670, 2001.

[15] E. Kohler, C. Keysers, M. A. Umilta, L. Fogassi, V. Gallese, and G. Rizzolati. Hearing sounds, understanding actions: action representation in mirror neurons. Science, (297), 846-848, 2002.

[16] A. Lahav, E. Saltzman, and G. Schlaug. Action Representation of Sound: Audiomotor Recognition Network While Listening to Newly Acquired Actions. The Journal of Neuroscience, 27(2), 308-314, 2007.

[17] B. Hommel. Action control according to TEC (theory of event coding). Psychological Research, 73, 512-526, 2009.

[18] M. Rath and D. Rocchesso. Continuous Sonic Feedback from a Rolling Ball. IEEE Multimedia, Special Issue on Interactive Sonification, 12(2), 60-69, 2005.

[19] L. Shams and A. R. Seitz. Benefits of multisensory learning. Trends in Cognitive Science, 12(11), 411-417, 2008.

[20] G. A. Calvert, M. J. Brammer, and S. D. Iversen. Crossmodal Identification. Trends in Cognitive Sciences, 2(7), 247-253, 1998.

[21] A. O. Effenberg and G. Schmitz. Multisensory motion perception: Influence of pitch modulation on the perception of movement velocity. In: F. Korkusuz, H. Ertan, and E. Tsolakidis (Eds.): Book of Abstracts of the 15th Annual Congress of the European College of Sport Science, Antalya, Turkey, 473, 2010.

[22] M. Müller. Information Retrieval for Music and Motion. Springer, 2007. 
[23] A. O. Effenberg. Movement sonification: Effects on perception and action. IEEE Multimedia, 12(2), 53-59, 2005.

[24] L. Scheef, H. Boecker, M. Daamen, U. Fehse, M. W. Landsberg, D. O. Granath, H. Mechling, and A. O. Effenberg. Multimodal motion processing in area V5/MT: Evidence from an artificial class of audio-visual events. Brain Research, 1252, 94-104, 2009.

[25] G. Schmitz, B. Mohammadi, A. Hammer, M. Heldmann, A. Samii, T. F. Münte, and A. O. Effenberg. Observation of sonified movements engages a basal ganglia frontocortical network.. Human Brain Mapping, in review. 